\title{
Artistic Interpretation of a Malaria Transmission Scenario
}

\author{
Vicky Isley, boredomresearch \\ Bournemouth University \\ Poole, UK \\ visley@bournemouth.ac.uk
}

\author{
Paul Smith, boredomresearch \\ Bournemouth University \\ Poole, UK \\ psmith@bournemouth.ac.uk
}

\begin{abstract}
Aggressive deforestation in Malaysia, motivated by a dramatic intensification of agriculture is currently of significant concern for its potential to destabilise interactions between species in ways that are significant to human health. Reports have emerged from Malaysia of naturally acquired human infections of a malaria parasite, Plasmodium knowlesi, previously thought only to infect long-tailed and pig-tailed macaques. In humans $P$. knowlesi has a rapid 24-hour replication cycle resulting in mortality from severe malaria. In response to a growing desire to understand the potential for spatially sensitive models of disease transmission and better understand the complex behaviour of infection transmission, the artist collaboration boredomresearch partnered with a mathematical modeller on an artistic expression which presents a malaria infection transmission scenario. The project combines tools and techniques from ecology, epidemiology, computer gaming and contemporary arts practice to explore this zoonotic malaria in Malaysia. This paper presents some of the insights and questions that arose from this collaboration to offer a view that encourages increased interaction across domains. Ultimately, highlighted are some potential distortions revealed during the transdisciplinary interaction.
\end{abstract}

Malaria. Computer animation. Disease transmission. Visual art. Computer graphics. Mathematical models.

\section{INTRODUCTION}

As part of an Animate Projects, Wellcome Trust funded commission, artists Vicky Isley and Paul Smith (boredomresearch), known for their computational and generative arts practice, began a collaboration with mathematical modeller $\mathrm{Dr}$ Paddy Brock. Based at the Institute of Biodiversity Animal Health and Comparative Medicine at the University of Glasgow, Brook is currently using analysis tools from ecology and epidemiology to explore zoonotic malaria in Malaysia. At the first meeting between artist and scientist Brock presented a simulation of disease transmission built using NetLogo (a multi-agent, programmable modeling environment). The simulation presented a black space populated by small graphic representations of humans who would change colour depending on their status; changing from susceptible, through infected, to infectious and hopefully ending at recovered. It later transpired that this animated visual expression was significantly different from the main body of Brock's practice that is normally expressed in the form of mathematical descriptions (Figure 1), intimidating to an untrained audience. It was for this reason that Brock had made the wise choice to present something more palatable to a visual-centric audience of artists.

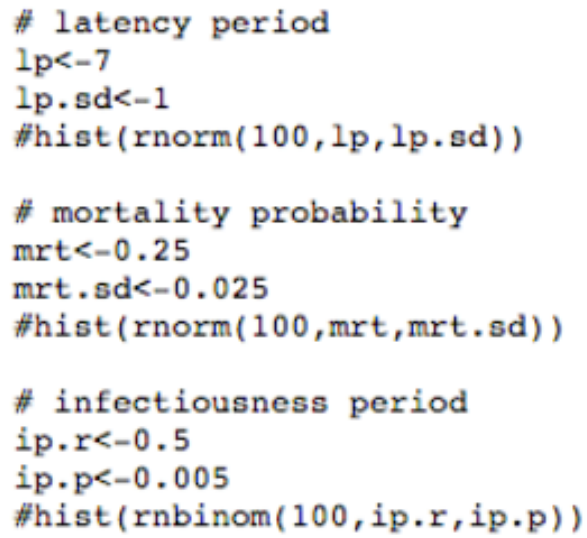

Figure 1: $R$ code from Brock, a stochastic SEIR (susceptible, exposed, infectious, recovered) model.

The work of boredomresearch is primarily created with computer code. They are fascinated with the mechanisms that give rise to the beauty that exists in the natural world and their practice seeks to explore this using the potential of contemporary computational technologies. For this reason, they 
were not daunted by the thought of a mathematical description. Their practice is often informed by such methods published in textbooks and scientific papers. In fact, they were hungry to gain a deeper understanding of the relationship between the abstract descriptions created by artists and those invented for scientific purposes. The early stages of the collaboration looked to unravel this conceptual space trying to understand the nature of this difference. At first, discussions were often confused as they negotiated the different language of arts and science, sometimes using words that had different meanings in each context or using different terms to describe what was, fundamentally, the same. Often, what was at first incomprehensible, would become suddenly familiar - sometimes superficially, sometimes deeply. Brock considering the work of boredomresearch noted:

\begin{abstract}
"Their work is similar...to the mathematical modelling of disease transmission; it uses similar software tools, but the inputs and outputs, incentives and insights, are quite different." (Brock 2014).
\end{abstract}

For example, in both cases the artists and scientist were working within the limitations of computing hardware making carefully considered economies that aim to respect the integrity of the desired outcome.

"Scientists try as hard as possible to make their mathematical models as close to reality as possible, but how often are they prevented from doing so by constraints of the model set-up, limitations of computer power etc. Is the distance that these uncontrollable constraints take the model away from a true representation of the real world further than the distance an artistic perspective would?" (Brock 2014)

From a visual arts perspective, boredomresearch found some scientific conventions challenging. For example, it is difficult to justify the use of a single coloured line to signify the deaths of millions of individuals. In many cases a mathematical description will not even represent individuals, instead a proportion of a population may be fit, unwell or dead. Often these models run invisibly in the depths of the computer only displaying a visual representation once all calculations have been made. Ultimately, it seems, these representations are devoid of individuals, space and time; qualities that boredomresearch felt were essential to form a deep appreciation of the subject under study. More recently, Brock has been interested in models of disease transmission that are spatially sensitive. This is relatively new in the field of epidemiology, that formerly favours the above described shapeless representations; devoid of individuals. Although, it was these highly abstract models, which informed much of the early discussions between artists and scientist.

Midway in the collaboration, Brock disappeared on a field trip to the Malaysian island of Banggi, to take part in the curiously named Monkeybar project. The Monkeybar project is a multidisciplinary collaborative research project on the zoonotic malaria, Plasmodium knowlesi. The project looks to define the biomedical, environmental and social risk factors for human infection with $\mathrm{P}$. knowlesi as well as the opportunities for prevention and control. Returning from Banggi island, Brock came to boredomresearch studio in December 2014 to share the latest work on this emerging zoonotic infection, $P$. knowlesi: a malaria parasite that was previously thought only to infect long-tailed and pigtailed macaques and discuss spillover into the human population. This interaction ultimately inspired the artwork AfterGlow (Figure 2) which combines 3D gaming technologies to present a highly aesthetic expression of disease transmission underpinned by mathematical models and land use data. The resulting work considers an infection transmission scenario primarily from a mosquito's perspective to express the complex interactions between human, landscape and infection.

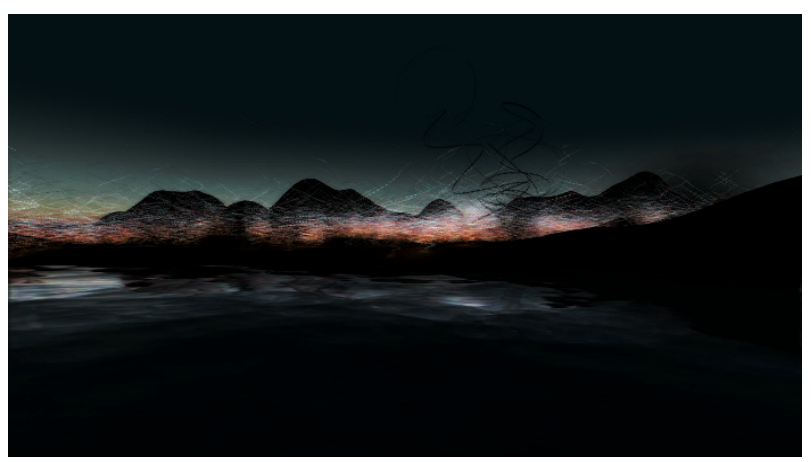

Figure 2: Screen capture image from AfterGlow, depicting edge of the island. (c) boredomresearch 2015.

AfterGlow is a 3D animated landscape that runs in real-time. Sixty times a second the model is updated and the results displayed on screen in the form of a landscape locked in perpetual twilight prime mosquito blood feeding time. Gradually the terrain becomes illuminated by glowing trails evocative of mosquito flight paths. These spiraling forms represent packets of blood infected with $P$. knowlesi as they are moved around by millions of infected mosquitoes. Animating the infection left in the wake of wandering macaques as they search the island for food, AfterGlow reveals the intimate relationship between disease and its environment. As an autonomous camera explores the dark mountains, the viewer becomes immersed in a blizzard of infection (Figure 3). Composed of delicate, spiraling, cells of colour, these combine to form a vivid expression of this dangerous disease. 


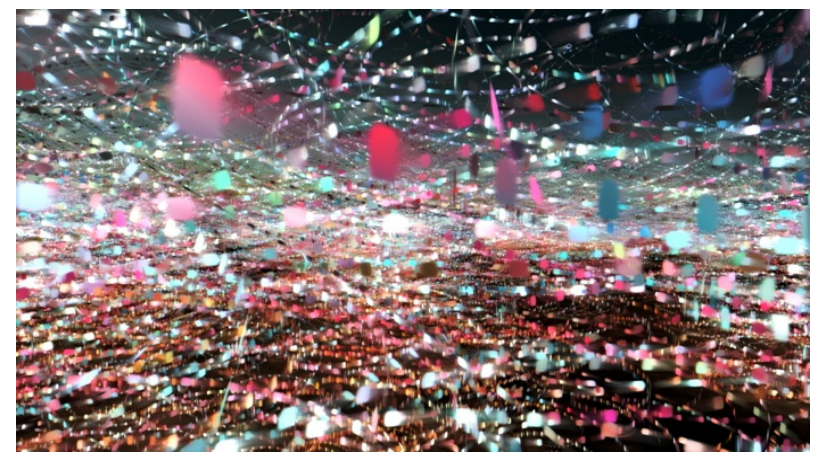

Figure 3: Screen capture image from AfterGlow, depicting blizzard of infection. (@) boredomresearch 2015.

The expression of disease transmission born out of this transdisciplinary approach is quite different to that which would be created in the service of science. The model continually updates without final conclusion. Here, emphasis is on the visual expression of a process; synchronous but abstracted from real time. This presents a completely different temporal experience of the models output. Unlike the abstract graphs and charts of science, the data takes a form immediately familiar to an audience accustomed to the moving image. Another difference of the artistic model from many scientific representations is an ambivalence towards a need for results. By avoiding the need to resolve a problem the artwork offers a frame of reference outside of orthodox scientific practice. In doing so the artist challenges the fundamental construct of scientific investigations, aimed at developing interventions, which are limited by narrow time frames imposed by funding cycles.

While the motive to reduce loss of life is rightly unworthy of challenge, it hides a graver concern that falls outside the timescale of a current frame of reference. This, more worrying concern is revealed by the reductive simplicity of the abstract mathematical description combined with a research project that considers ecology and epidemiology. A few simple changes can eradicate the problem of zoonotic malaria. No forest - no macaques - no malaria. In terms of disease control, do the longterm goals sit comfortably with desires to promote and maintain a stable co-existence of species? Agustin Fuentes in considering the current management of human/macaque interfaces documents the primary approaches to reduce conflict: "modifying the environments in which humans and macaque come into contact"; changing "the composition and behavior of macaque populations"; and "modifying the composition and or behavior of human populations" (Fuentes 2011). From these studies the success and integrity of past and existing management programs is questionable. What is clear is a strong tendency toward controlling macaque population size.

The ultimate question is whether the current frame of reference, limited by the narrow focus of current mathematical descriptions, is supporting a distorted worldview that will ultimately cause greater harm in the longer term.

\section{USING SCIENTIFIC DATA BUT NOT AS AN ACCURATE MODEL}

During the collaboration with Brock boredomresearch were keen to better understand what makes a scientific model or representation useful, accurate and meaningful. How these models are evaluated and how they might differ from those motivated by an artistic expression of the subject.

In the case of malaria, one example of usefulness could be understood as an ability to facilitate a productive intervention against a backdrop of complex ethical, political and economic considerations. These are not necessarily incorporated in the mathematical description itself but are often important factors for general consideration. More complex environmental considerations are often marginalised. The required paradigm shifts to change some broader global beliefs, appear out of reach. Therefore, the usefulness of a model is judged in terms of welldefined but less challenging goals. An illustration of this is the focus on body count. Statistics like "Globally, the disease caused an estimated 453,000 under-five deaths in 2013" (WHO 2014) is an example of a tendency to reduce the evaluation of productivity to one single factor, which despite its undeniable importance, also introduces a significant emotional bias. More importantly it introduces a bias to the present, ignoring more farreaching implications of further destabilisation of complex ecological interactions - inducing potentially more severe consequences for future generations. Seemingly robust arguments, primarily supported in terms of human cost, fail to consider a long-term future on a potentially destabilised planet. Human cost is reduced to a league table, with a percentage increase in lives saved from the previous year. This short sightedness is acceptable primarily because the lives of future generations seem to be valued based on their proximity to the present. We lack the ability to value the lives of those who will not be born for another thousand years, who seem as unworthy of our concern as the nameless millions that have already been dead a millennium. We are aware, maybe, that the deeper we project our concerns into the future the more inaccurate they become? 
Accuracy, surely, is the bedrock of science. What sane scientist would advertise that their findings were inaccurate? Surely inaccuracies are the flawed components that bring theories crashing down. boredomresearch wanted to understand if an artwork could incorporate an accurate description of an infection scenario. In discussion with their scientific collaborator, seemingly straightforward concepts of accuracy became unclear. They began to fog a widely-held assumption that science presents accurate, substantiated truth in contrast to the subjectivity of the arts.

\section{"When boredomresearch talk about an artistic description of an imagined place, it brings to mind hidden process models, and the over- looked creativity that is an integral part of scientific hypothesis generation." (Brock 2014).}

As we have seen, mathematical descriptions tend to be highly reductive, attempting to represent a scenario in an eloquent and interpretable form. They are often based on fieldwork, that is at best patchy, and have to accommodate voids in both understanding and data. These voids are often filled by creative invention. Kleinschmidt et al. considering a spatial statistical approach to malaria mapping stating that: "A concern with spatial data is the potential for spatial correlation in the observations, which could lead to incorrect estimates" and attempts to identify techniques to accommodate the "spatial clustering of disease" that is almost inevitable as "human populations generally live in spatial clusters" (Kleinschmidt et al. 1999). In addition to inaccuracies or distortions arising from such insufficiencies, scientific models are deliberately vague. This relates to a notion of generalisability, where in order to be applicable across a broad domain, non-specificity is required. A specific insight is limited to a specific case as opposed to one that is generally applicable and broadly valuable. Here science is inaccurate by design. Artistic representations often favour a direct link with a specific individual, case or location but this does not make them completely unrelated to scientific practice.

\footnotetext{
"This process of eliminating unnecessary information while retaining the integrity of an idea or thing is a step towards greater generality. Once an artist or scientist has found a powerful abstraction, he or she naturally wants to know how generally it can be applied. This process involves pattern recognition, the ability to organize the random events we see, hear, or feel by grouping them." (Root-Bernstein et al. 2004).
}

This profoundly contradicts the former oversimplification that science offers accuracy absent in the arts. By challenging these fundamental beliefs, which infer disciplines in opposition, we provide a valuable opening to interact meaningfully across established boundaries. The abstract computational representation of disease transmission employed in AfterGlow is distinctly different from that which would satisfy the needs of a scientific investigation but it is in no way less accurate. The work gives visual form to the incomprehensible complexity of a mechanism that employs unknown billions of agents in its manifestation. This is distinctly different from a result in terms of human cost, or death toll, but very relevant in respect to a growing cultural understanding of our relation to and interaction with complex environmental mechanisms.

For the authors, the most rewarding concept to consider from both an artistic and scientific perspective is that of meaningfulness. Already, we have seen that some of the most basic assumptions around scientific integrity are less than straight- forward but it is when considering what might constitute a meaningful expression that we may struggle to negotiate the space between arts and science. Much of scientific endeavour is funded, regulated, interrogated and evaluated independently from mainstream contemporary culture. These firms, agencies and institutions are often invisible in the public domain. That is not to say that activity is not mediated, presented and ultimately implemented in the public domain but as soon as we question the meaningfulness of any endeavour we need to consider a more complete and comprehensive evaluation.

In the above, illustrated collaboration, questions have been raised that suggest a worrying temporal distortion implicit in the scientific process. It is clear that the potential ramifications are both significant and increasingly relevant in a world subject to accelerating human interaction. It is also possible that greater cultural interaction may provide valuable societal tools to deal with complex ecological concerns. Organisations like the Wellcome Trust are recognised for their "purposeful entertainment, creative collaborations and fresh insight on both science and art" and it is noted that: "the oblique nature of an artwork can sometimes illuminate a deeply sensitive and difficult scientific topic" (Caines 2014) but there is much more that the arts should offer to interrogate and make sense of the scientific realm.

It is within a collaborative project like that of AfterGlow that the authors could open a dialogue between distinct and separate fields of practice. As part of this cultural evaluation, they present a visual expression of a malaria infection transmission scenario that considers the as yet incomprehensible complexity of the natural world. AfterGlow may omit incredibly important dimensions relating to human health, focusing primarily on a scenario from a mosquito's 
perspective but it does address the mind-boggling complexity of a real life environment bursting with billions of mosquitoes, each carrying a packet of infected blood. The artwork does not finish and provide a graph of its results from which urgent decisions can be made, preferring to challenge a technologically induced tendency towards immediacy. Moving beyond the ever expansive now, we do not deliver results in the quickest possible time but create an expression of disease transmission that is experienced over time. We hope that in so doing we provide a conceptual extension that may help bridge the gap between the domains of nature, science, technology and society.

\section{REFERENCES}

Caines, M. (2014) Arts head: Lisa Jamieson, head of engaging science, the Wellcome Trust. The Guardian, 4 November.

https://www.theguardian.com/culture-professionalsnetwork/2014/nov/04/engaging-science-wellcometrust-arts-head (retrieved 22 March 2017).
Gumert, M., Fuentes, A., and Jones-Engel, L. (2011) Monkeys on the Edge: Ecology and Management of Long-Tailed Macaques and their Interface with Humans. Cambridge University Press, UK.

Kleinschmidt, I., Bagayoko, M., Clarke, G. P. Y., Craig, M., and Le Sueura, D. (2000) A spatial statistical approach to malaria mapping. International Journal of Epidemiology, 29(2), pp. 355-361.

Root-Bernstein, R. and Root-Bernstein, M. (2004) Artistic Scientists and Scientific Artists: The Link Between Polymathy and Creativity. In Sternberg, R. J., Grigorenko, E. L., \& Singer, J. L. (eds.), Creativity: From Potential to Realization. American Psychological Association, Washington, DC.

WHO (2014) World Malaria Report. Factsheet on the World Malaria Report. World Health Organisation, Geneva, Switzerland.

http://www.who.int/malaria/publications/world mala ria report 2014/en/ (retrieved 21 May 2017). 